\title{
ASTROPHYSICAL NEUTRINOS: 20th CENTURY AND BEYOND
}

\author{
J. N. BAHCALL, IUPAP Centennial Lecturer ${ }^{\mathrm{a}}$ \\ ${ }^{a}$ Institute for Advanced Study, Princeton, NJ 08540, USA \\ E-mail: www.sns.ias.edu/ jnb
}

I summarize the first four decades of solar neutrino research and suggest what may be possible to learn with extragalactic neutrinos and with solar neutrinos in the next decade.

\section{Introduction}

I was asked by Art McDonald to give one of the opening talks on the subject " Neutrino Astrophysics in the 20th Century and Beyond." Feeling very honored, I readily accepted. But, as I started thinking about what I should say to so many knowledgeable friends, I got really worried. I realized that it would be impossible in twentyfive minutes to discuss intelligently all of neutrino astrophysics of the 20th century. There is just too much important material to cover even if I spoke very fast, unintelligibly fast, and even if I did not say anything about our goals for the future.

So, I decided to limit my remarks to two specific topics: solar neutrinos and extragalactic neutrinos. I will not say anything about the enormous achievements in the prediction and detection of supernova neutrinos and in the calculations of neutrino cooling processes for stars that are not exploding. I will also not discuss the role of neutrinos in Big Bang nucleosynthesis nor in cosmology. There are lots of grand things to say about these subjects, and many other topics in neutrino astrophysics, but I will not discuss them today.

I will take a somewhat historical approach and emphasize those aspects of the development of our subject which may help guide our thinking about what we should do in the future. I will begin with solar neutrinos and then switch abruptly to extragalactic neutrinos.

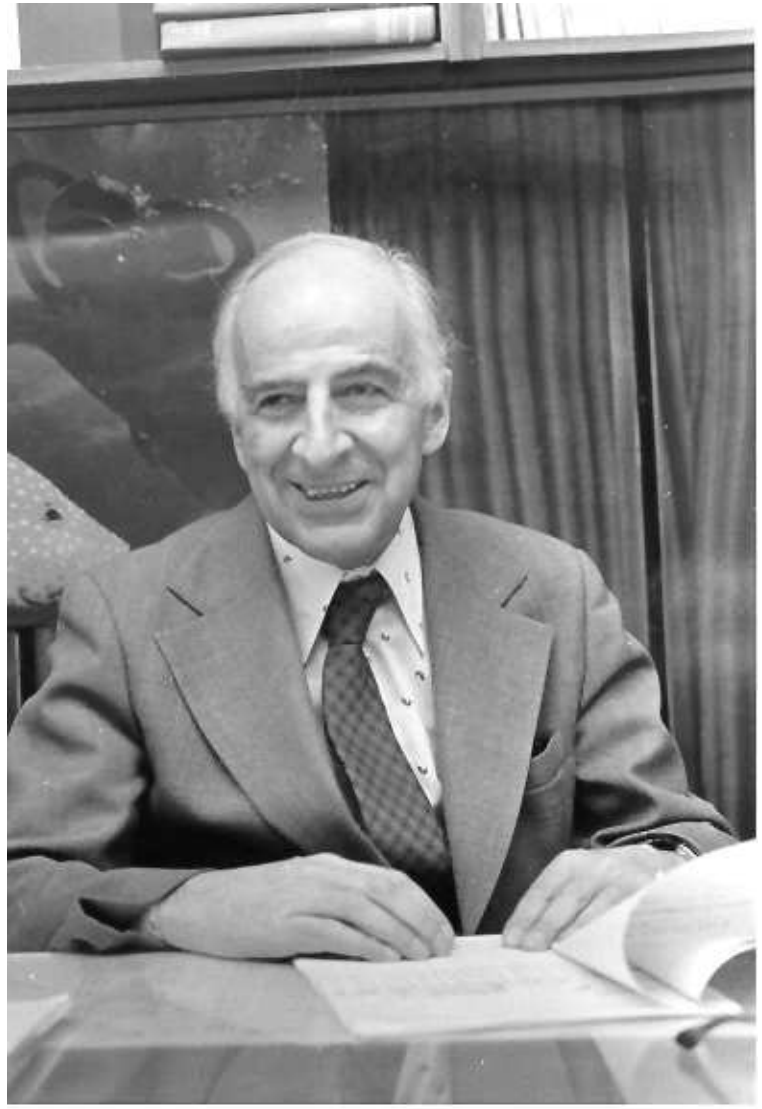

Figure 1. Bruno Pontecorvo wrote in 1967: 'From the point of view of detection possibilities, an ideal object is the sun.' Figure courtesy of S. Bilenky . 


\section{Solar neutrinos}

\subsection{Bruno Pontecorvo and Ray Davis}

I want to begin by paying tribute to two of the great scientists and pioneers of neutrino astrophysics, Ray Davis and Bruno Pontecorvo. Bruno first suggested using chlorine as a detector of neutrinos in a Chalk River report written in 1946. Ray followed Bruno's suggestion and the careful unpublished feasibility study of Louie Alvarez. Using with care and skill a chlorine detector and reactor neutrinos, Ray showed in 1955-1958 that $\nu_{e}$ and $\bar{\nu}_{e}$ were different. About a decade later, Ray first detected solar neutrinos, laying the foundation for the studies we shall hear about today.

In 1967, one year before the first results of Ray's chlorine solar neutrino experiment were announced, Bruno published a prophetic paper entitled: 'Neutrino Experiments and the Problem of Conservation of Leptonic Charge' [Zh. Exp. Teor. Fiz. 53, 1717 (1967)]. In this paper, Bruno suggested many different experiments that could test whether leptonic charge was conserved. The grandchildren of most of these experiments are being discussed in this conference, Neutrino 2000.

Bruno included a short section in his paper that he called 'Oscillations and Astronomy.' In this section, Bruno wrote: "From the point of view of detection possibilities, an ideal object is the sun," What a wonderfully contemporary statement!

Bruno, like most particle physicists of the 1960 's and perhaps 1970's and 1980's, did not believe astrophysical calculations could be reliable. He wrote in this same section on oscillations and astronomy: "Unfortunately, the weight of the various thermonuclear reactions in the sun, and the central temperature of the sun are insufficiently well known in order to allow a useful comparison of expected and observed solar neutrinos, from the point of view of this article." [This was 30 years before the precise confirmation of the standard solar model by helioseismology.] To support his claim, Bruno referenced only his 1946 Chalk River report, which mentioned the sun in just two sentences. Bruno did cite our calculations of the solar neutrino fluxes elsewhere in his 1967 paper, but they seem not to have affected his thinking.

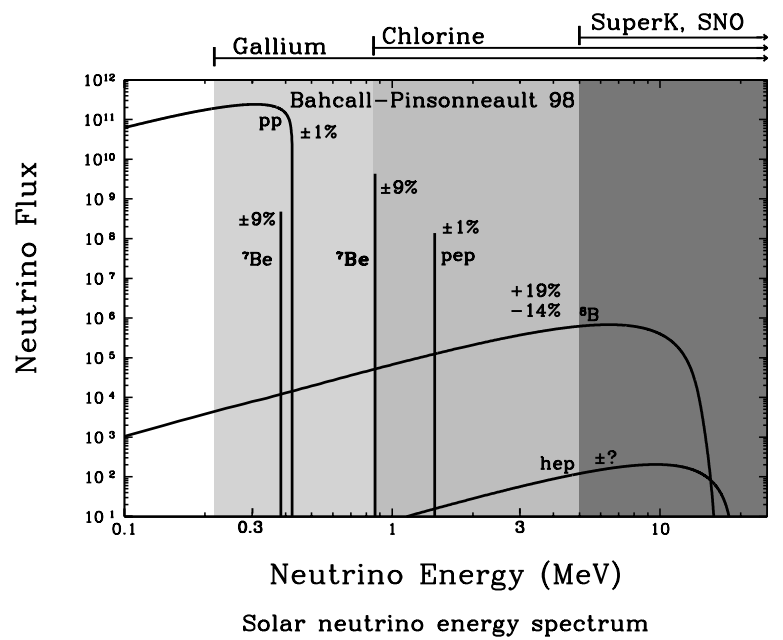

Figure 2. The energy Spectrum of neutrinos from the pp chain of interactions in the Sun, as predicted by the standard solar model. Neutrino fluxes from continuum sources (such as $p-p$ and ${ }^{8} \mathrm{~B}$ ) are given in the units of counts per $\mathrm{cm}^{2}$ per second. The percentage errors are the calculated $1 \sigma$ uncertainties in the predicted fluxes. The $p-p$ chain is responsible for more than $98 \%$ of the energy generation in the standard solar model. Neutrinos produced in the carbon-nitrogen-oxygen $\mathrm{CNO}$ chain are not important energetically and are difficult to detect experimentally. The arrows at the top of the figure indicate the energy thresholds for the ongoing neutrino experiments. This spectrum is from BP98: J. N. Bahcall, S. Basu, and M. H. Pinsonneault, Phys. Lett. B, 433, 1 (1998).

What can we learn from this bit of history? When Ray and I wrote our PRL papers arguing that a chlorine detector of 600 tons could observe solar neutrinos, we never discussed the possibility of using neutrinos to learn about particle physics. The only motivation we gave was "...to see into the interior of a star and thus verify directly the hypothesis of nuclear energy generation in stars." [PRL 12, 300 (1964)].

Why did we not discuss using neutrinos for particle physics? Frankly, because we never thought 
about it. And even if we had, we would have known better than to mention it to our particle physics friends. Bruno had the insight and the vision and indeed the courage to argue that astronomical neutrinos could potentially give us unique information about neutrino characteristics. His paper is all the more remarkable because it was published a year before the first results of the chlorine experiment showed that the rate Ray observed was less than our calculated rate.

We learn from these events that pioneering experiments can lead to important results in areas that are unanticipated. We will come back to this conclusion at the end of this talk.

\subsection{Standard Model Predictions}

Figure 2 shows the calculated solar neutrino spectrum predicted by the Standard solar model. The percentage errors are the calculated $1 \sigma$ uncertainties in the predicted fluxes, based upon the published errors of the measured quantities and on many calculations of standard solar models. As you will hear from the talks in the later parts of this morning session, the total intensities and the energy spectra shown in Fig. 2 are now widely used to interpret, and indeed to plan, solar neutrino experiments such as those discussed in today's sessions: chlorine, Super-Kamiokande, SNO, SAGE, GALLEX, GNO, and BOREXINO.

Figure 3 compares the calculated versus the measured rates for the six solar neutrino experiments for which results have been reported. Assuming nothing happens to the neutrinos after they are created, the measured rates range from $33 \% \pm 5 \%$ of the calculated rate (for chlorine) to $58 \% \pm 7 \%$. As is now well known, the observed rates cannot be fit (at a C.L. of about $99 \%$ ) with any linear combination of undistorted solar neutrino energy spectra.

Today we know that there are three reasons that the calculations of solar neutrino fluxes are robust: 1) the availability of precision measurements and precision calculations of input data that have been gradually refined over four decades; 2) the intimate connection between neutrino fluxes and the measured solar luminosity; and 3) the measurement of the helioseismological frequencies of the solar pressure-mode ( $p$-mode) eigenfrequencies.

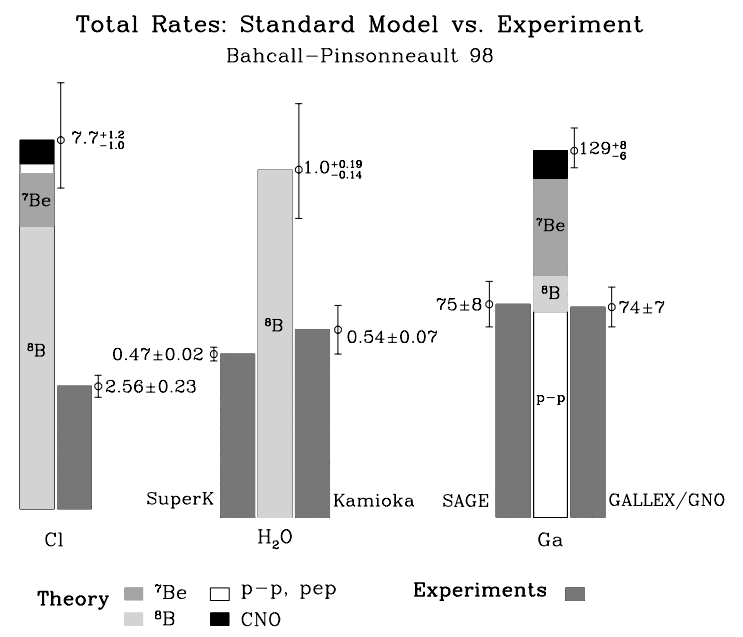

Figure 3. Comparison of measured rates and standard-model (BP98) predictions for six solar neutrino experiments. The unit for the radiochemical experiments (chlorine and gallium) is $\mathrm{SNU}\left(10^{-36}\right.$ interactions per target atom per sec); the unit for the water-Cerenkov experiments (Kamiokande and Super-Kamiokande) is the rate predicted by the standard solar model plus standard electroweak theory. The experimental results are described by Lande, Suzuki, Gavrin, and Belotti in these proceedings.

Could the solar model calculations be wrong by enough to explain the discrepancies between predictions and measurements shown in Fig. 3? Helioseismology, which confirms predictions of the standard solar model to high precision, suggests that the answer is "No."

Figure 1 shows the fractional differences between the most accurate available sound speeds measured by helioseismology and sound speeds calculated with our best solar model (with no free parameters). The horizontal line corresponds to the hypothetical case in which the model predictions exactly match the observed values. The rms fractional difference between the calculated and the measured sound speeds is $1.1 \times 10^{-3}$ for 
the entire region over which the sound speeds are measured, $0.05 R_{\odot}<R<0.95 R_{\odot}$. In the solar core, $0.05 R_{\odot}<R<0.25 R_{\odot}$ (in which about $95 \%$ of the solar energy and neutrino flux is produced in a standard model), the rms fractional difference between measured and calculated sound speeds is $0.7 \times 10^{-3}$.

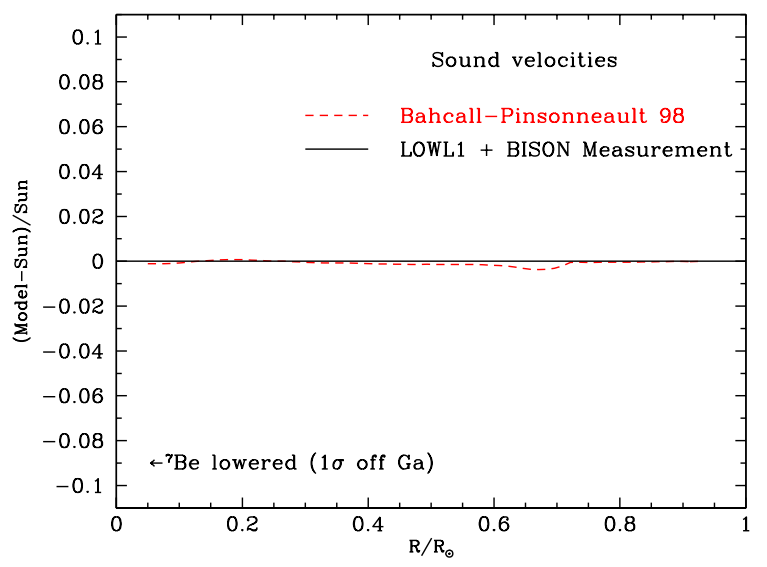

Figure 4. Predicted versus Measured Sound Speeds. This figure shows the excellent agreement between the calculated (solar model BP98, Model) and the measured (Sun) sound speeds, a fractional difference of $0.001 \mathrm{rms}$ for all speeds measured between $0.05 R_{\odot}$ and $0.95 R_{\odot}$. The vertical scale is chosen so as to emphasize that the fractional error is much smaller than generic changes in the model, 0.09, that might significantly affect the solar neutrino predictions. The measured sound speeds are from S. Basu et al., Mon. Not. R. Astron. Soc. 292, 234 (1997); The figure is taken from BP98.

The arrow in Fig. 4 shows how different the solar model sound speeds would have to be from the observed sound speeds if one wanted to use solar physics to reduce the ${ }^{7}$ Be neutrino flux. The position of the arrow is fixed by artificially reducing the predicted ${ }^{7} \mathrm{Be}$ neutrino flux that is not observed in the gallium experiments, SAGE and GALLEX plus GNO (see Fig. 3). if the $p-p$ neu- trinos are present. Remember, we believe we can calculate the $p-p$ flux to $\pm 1 \%$.

The discrepancy with the hypothetical new solar physics was estimated by using the temperature dependence of the ${ }^{7}$ Be neutrino flux $\left(\propto T^{10}\right)$ and the sound speeds $\left(\propto T^{1 / 2}\right)$. The agreement with the hypothetical solar physics is more than 100 times worse than the agreement with the Standard Model physics.

Figure 4 has contributed to the consensus view that the experimental results shown in Fig. 3 require new particle physics for their explanation.

\subsection{Summing up and looking ahead}

I want now to look back and then look ahead. I will begin by giving my view of the principal accomplishments of solar neutrino research to date (Sect. 2.3.1). Then I will discuss two of the expected highlights of the next decade of solar neutrino research, the measurement of the neutral current to charge current ratio for ${ }^{8} \mathrm{~B}$ neutrinos ((Sect.2.3.2) and the detection of solar neutrinos with energies less than $1 \mathrm{MeV}$ ((Sect. 2.3.3).

\subsubsection{Principal achievements}

What are the principal achievements of the first four decades of solar neutrino research? I give below my personal list of the 'top three achievements.'

- Solar neutrinos have been detected. The chlorine, Kamiokande, Super-Kamiokande, GALLEX, SAGE, GNO, and SNO experiments have all measured solar neutrino events. This is the most important achievement. The detection of solar neutrinos shows empirically that the sun shines by the fusion of light elements.

- Evidence for new physics has been found. For more than thirty years, beginning with the fact that Ray's first measurements in 1968 indicated a flux lower than the standard model predictions, we have had evidence for new physics in the solar neutrino arena. This evidence has steadily deepened as new solar neutrino experiments have confirmed and extended the neutrino discrepancies and helioseismology has confirmed the standard solar model. The fact that neutrino oscillations have now been observed in atmospheric neutrino phenomena fur- 
ther strengthens the case that oscillations occur for solar neutrinos. We are still looking for a 'smoking gun' single effect that shows up in just one solar neutrino experiment, rather than combining the results of two or more different experiments. I will discuss some possibilities below.

- Neutrino fluxes and energy spectra are approximately as predicted by the standard solar model. If you had told me in 1964 that six solar neutrino experiments would give results within a factor of three of the predicted standard model results, I would have been astonished and delighted. This is especially so considering that the crucial ${ }^{8} \mathrm{~B}$ neutrino flux depends upon the 25th power of the central temperature of the sun. This agreement exists without making any corrections for neutrino oscillations.

If we correct the observed solar neutrino event rates for the effects of neutrino oscillations using the six currently allowed two-neutrino oscillation scenarios, the inferred ${ }^{8} \mathrm{~B}$ neutrino flux at the source is rather close to the best-estimate predicted flux. At the $99 \%$ CL, one infers (see hep$\mathrm{ph}$ (9911248):

$0.55 \leq \phi\left({ }^{8} B\right) /($ Standard prediction $) \leq 1.32$

which is a slightly tighter range than the $3 \sigma$ prediction of the standard solar model.

\subsubsection{SNO and the $[\mathrm{NC}] /[\mathrm{CC}]$ ratio}

Figure 5 shows the predictions of the currently allowed neutrino oscillation solutions for the double ratio, $[\mathrm{NC}] /[\mathrm{CC}]$, of neutral current to charged current event rates in the deuterium detector SNO. Art McDonald will describe later this morning the experimental characteristics of this great observatory and outline for us the extensive program of SNO measurements. The important message of Fig. 同 is that all of the currently allowed oscillation solutions for active neutrinos predict a value for the double ratio that is different from the no oscillation value of 1.0 by at least nine times the estimated non-statistical measurement uncertainty.

We all eagerly look forward to this crucial and decisive measurement.

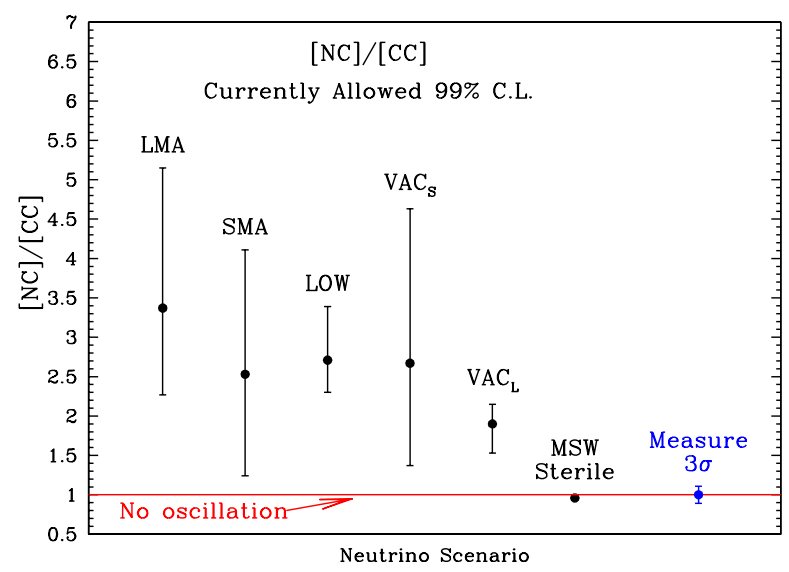

Figure 5. The neutral current to charged current double ratio, $[\mathrm{NC}] /[\mathrm{CC}]$. The standard model value for $[\mathrm{NC}] /[\mathrm{CC}]$ is 1.0 . The figure shows, for a $5 \mathrm{MeV}$ threshold for the $\mathrm{CC}$ measurement, the predicted double ratio of Neutral Current to Charged Current for different neutrino scenarios. The solid error bars represent the $99 \%$ C.L. for the allowed regions of the six currently favored neutrino oscillation solutions. The dashed error bar labeled "Measure $3 \sigma$ " represents the net estimated uncertainty in interpreting the measurements, including the energy resolution, energy scale, ${ }^{8} \mathrm{~B}$ neutrino energy spectrum, neutrino cross section, counting statistics, and the hep flux. This is Fig. 7a of Bahcall, Krastev, and Smirnov, hep-ph/0002293.

\subsubsection{Solar neutrinos below $1 \mathrm{MeV}$}

More than $98 \%$ of the calculated standard model solar neutrino flux lies below $1 \mathrm{MeV}$. The rare ${ }^{8} \mathrm{~B}$ neutrino flux is the only solar neutrino source for which measurements of the energy have been made, but ${ }^{8} \mathrm{~B}$ neutrinos constitute a fraction of less than $10^{-4}$ of the total solar neutrino flux.

The great challenge of solar neutrino astronomy is to measure neutrino fluxes below $1 \mathrm{MeV}$. We must develop experiments that will measure the ${ }^{7} \mathrm{Be}$ neutrinos (energy of $0.86 \mathrm{MeV}$ ) and the fundamental $p-p$ neutrinos $(<0.43 \mathrm{MeV})$. A number of promising possibilities were discussed at the LowNu workshop that preceded this confer- 
ence. The BOREXINO observatory, which can detect $\nu-e$ scattering, is the only approved solar neutrino experiment which can measure energies less than $1 \mathrm{MeV}$.

The $p$ - $p$ neutrinos are overwhelmingly the most abundant source of solar neutrinos, carrying about $91 \%$ of the total flux according to the standard solar model. The ${ }^{7} \mathrm{Be}$ neutrinos constitute about $7 \%$ of the total standard model flux.

We want to test and to understand neutrino oscillations with high precision using solar neutrino sources. To do so, we have to measure the neutrino-electron scattering rate with ${ }^{7} \mathrm{Be}$ neutrinos, as will be done with the BOREXINO experiment, and also the CC (neutrino-absorption) rate with ${ }^{7} \mathrm{Be}$ neutrinos (no approved experiment). With a neutrino line as provided by ${ }^{7} \mathrm{Be}$ electroncapture in the sun, unique and unambiguous tests of neutrino oscillation models can be carried out if one measures both the charged-current and the neutral current reaction rates.

I believe that we have calculated the flux of $p-p$ neutrinos produced in the sun to an accuracy of $\pm 1 \%$. This belief should be tested experimentally. Unfortunately, we do not yet have a direct measurement of this flux. The gallium experiments only tell us the rate of capture of all neutrinos with energies above $0.23 \mathrm{MeV}$.

The most urgent need for solar neutrino research is to develop a practical experiment to measure directly the $p-p$ neutrino flux and the energy spectrum of electrons produced by weak interactions with $p-p$ neutrinos. Such an experiment can be used to test the precise and fundamental standard solar model prediction of the $p-p$ neutrino flux. Moreover, the currently favored neutrino oscillation solutions all predict a strong influence of oscillations on the low-energy flux of $\nu_{e}$.

Figure 6 shows the calculated neutrino survival probability as a function of energy for three global best-fit MSW oscillation solutions. You can see directly from this figure why we need accurate measurements for the $p-p$ and ${ }^{7} \mathrm{Be}$ neutrinos. The currently favored solutions exhibit their most characteristic and strongly energy dependent features below $1 \mathrm{MeV}$. Naturally, all of the solutions give similar predictions in the en-

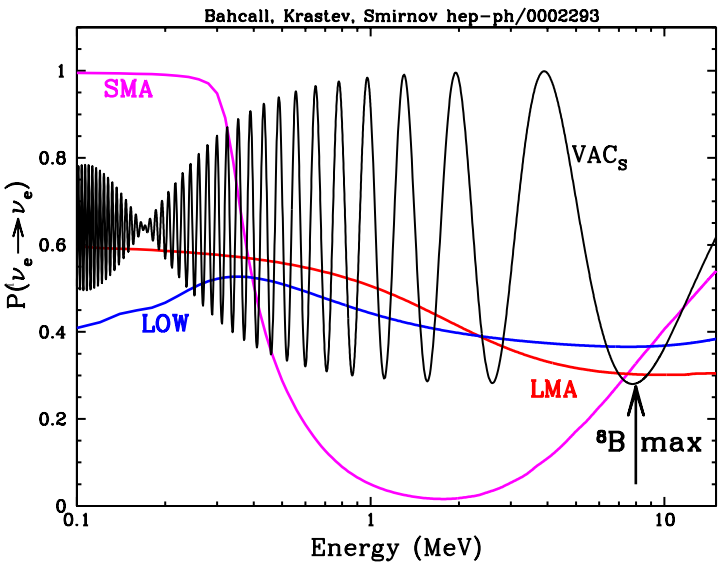

Figure 6. Survival probabilities for MSW solutions. The figure presents the yearly-averaged survival probabilities for an electron neutrino that is created in the sun to remain an electron neutrino upon arrival at the Super-Kamiokande detector.

ergy region, $\sim 7 \mathrm{MeV}$, where the Kamiokande and Super-Kamiokande data are best. The survival probability shows a strong change with energy below $1 \mathrm{MeV}$ for all the solutions, whereas in the region above $5 \mathrm{MeV}$ (accessible to SuperKamiokande and to SNO) the energy dependence of the survival probability is at best modest.

The $p$ - $p$ neutrinos are the gold ring of solar neutrino astronomy. Their measurement will constitute a simultaneous and critical test of stellar evolution theory and of neutrino oscillation solutions.

\section{Extragalactic neutrinos}

Experimentalists often like to describe the power of their experiments in terms of the expected or observed number of events per year and $L / E$, where $L$ is the distance between the accelerator and the detector and $E$ is the beam energy. The quantity $L / E$ determines, together with the square of the mass difference, the survival probability for vacuum neutrino oscillations. More generally, $L / E$ represents the time of flight in the rest 


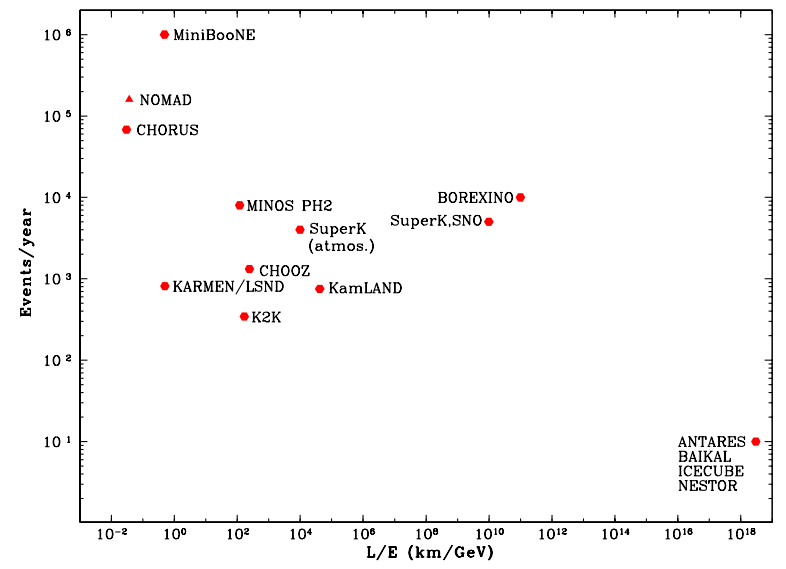

Figure 7. Very longbaseline neutrino oscillation experiments. The figure shows that experiments such as ANTARES, BAIKAL, ICECUBE, and NESTOR, which may detect high-energy neutrinos from distant gamma-ray bursts, have extraordinary sensitivity to vacuum neutrino oscillations. Neutrinos of $10^{5}$ $\mathrm{GeV}$ from gamma-ray bursts located at cosmological distances were used to locate the positions of ANTARES, BAIKAL, ICECUBE, and NESTOR in the figure.

frame of the particle, the time for rare events to occur.

Figure 7 shows the extraordinary sensitivity to neutrino oscillation of experiments like ANTARES, BAIKAL, ICECUBE, and NESTOR that can detect neutrinos from distant extragalactic sources. The accelerator experiments that will be discussed at Neutrino 2000 lie in the left-hand side of Fig. 6, $L / E<10^{4} \mathrm{~km} / \mathrm{GeV}$. Solar neutrino experiments like Super-Kamiokande, SNO, and BOREXINO can reach to $10^{10} \mathrm{~km} / \mathrm{GeV}$ and, for the lower energy experiments, even $10^{11}$ $\mathrm{km} / \mathrm{GeV}$. Extragalactic sources such as gammaray-bursts (GRBs) have such a long baseline $(\sim$ $10^{10}$ lyrs) that the new generation of extragalactic experiments, ANTARES, BAIKAL, ICECUBE, and NESTOR will extend to the right-hand side of Fig. 7, to $L / E>10^{18} \mathrm{~km} / \mathrm{GeV}$.

I want to say a few words about the possi- bilities for detecting GRB neutrinos, which will be discussed in more detail in these proceedings by Eli Waxman. I believe that GRBs offer the best chance for detecting extragalactic neutrinos among all the known sources of astronomical photons.

The phenomenology of the photons observed from gamma-ray bursts is now relatively well understood. Many different types of observations have been carried out and the results are well summarized by the expanding fireball model. Using this model, one can work out the flux of neutrinos from shocks.

Figure 8 shows the neutrino energy spectra that Waxman and I have estimated to be produced by GRBs, both from the direct burst (energies $10^{6} \mathrm{GeV}$ ) and from the afterglow (energies $\sim 10^{8}$ $\mathrm{GeV}$ to $\sim 10^{19} \mathrm{GeV}$ ). The observed population of GRBs should give rise to $\sim 10$ events per $\mathrm{km}^{2}$ per year from neutrinos with characteristic energies of order $10^{14} \mathrm{eV}$. We shall hear on the last day of this conference that the calculated GRB flux may be detectable in ANTARES, ICECUBE, or NESTOR. The fundamental assumption used in calculating the GRB neutrino flux is that GRBs produce the observed flux of high-energy cosmic rays, an assumption for which Eli Waxman has provided a strong plausibility argument.

GRBs occur at modest to large redshifts. We know the time of the explosion to an accuracy $\sim 10 \mathrm{sec}$ (from the gamma rays). Therefore, GRBs can be used to test special relativity to an accuracy of 1 part in $10^{16}$ and to test the weak equivalence principle to an accuracy of 1 part in $10^{6}$. If special relativity is right, the photons and the neutrinos should arrive at the same time (to an accuracy of about $10 \mathrm{sec}$, the duration of the burst). If the weak equivalence principle is valid, the arrival times of neutrinos (which traverse significant gravitational potentials) from distant sources should be independent of neutrino flavor.

GRBs can also be used to probe the weak interactions to an extraordinary level of precision. Gamma-ray bursts are expected to produce only $\nu_{e}$ and $\nu_{\mu}$. The large area detectors of extragalactic neutrinos are in principle sensitive to vacuum neutrino oscillations with mass differences as 


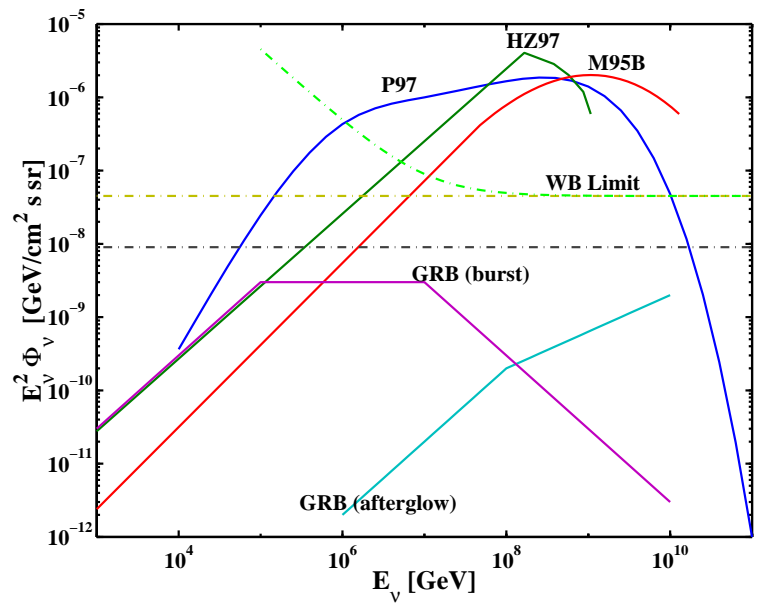

Figure 8. The Waxman-Bahcall upper bound on muon neutrino intensities $\left(\nu_{\mu}+\bar{\nu}_{\mu}\right)$. This figure is from Bahcall and Waxman, hep-ph/9902383. The numerical value of the bound assumes that $100 \%$ of the energy of protons is lost to $\pi^{+}$and $\pi^{0}$ and that the $\pi^{+}$all decay to muons that also produce neutrinos. The dot-dash line gives the upper bound corrected for neutrino energy loss due to redshift and for the maximum known evolution (QSO or star-formation evolution). The lower line is obtained assuming no evolution. The solid curves show the predictions of representative AGN jet models taken from the earlier papers of Mannheim (marked M95B in the figure), Protheroe (P97), and Halzen and Zas (HZ97). The AGN models were normalized so that the calculated gamma-ray flux from $\pi^{0}$ decay fits the observed gamma-ray background.

small as $\Delta m^{2} \geq 10^{-17} \mathrm{eV}^{2}$ (from $\nu_{\mu} \rightarrow \nu_{\tau}$ ).

Not everything is encouraging in Fig. 8. The figure also shows the upper limit that is allowed for astrophysical neutrino production from $(\gamma, \pi)$ interactions on high energy protons. The upper limit is established by using the observed cosmic ray flux of high energy protons. Prior to the recognition of this limit a number of authors had suggested much more optimistic models (also shown in the figure), that were normalized by fit- ting $\pi^{0}$ decay to the observed gamma-ray background.

\section{Goals for Astrophysical neutrinos: 2000-2010}

It seems to me that we have three principal goals for this next decade.

- Determine the mixing angles and mass differences that are important for solar neutrino phenomena.

- Test precisely stellar evolution by observing $p-p$ and ${ }^{7} \mathrm{Be}$ neutrinos, and by determining the total flux of ${ }^{8} \mathrm{~B}$ neutrinos.

- Discover extragalactic neutrinos, perhaps from gamma-ray bursts.

From time to time, friends ask me to compare the search for solar neutrinos with the search for neutrinos from GRBs. They are very different. From photon studies, we know more observationally about the sun than about any other astronomical source, certainly much more than about the mysterious GRBs. Moreover the sun is in the simplest stage of stellar evolution, in quasi-static equilibrium with a characteristic time scale for evolution of $10^{9} \mathrm{yr}\left(10^{16} \mathrm{~s}\right)$. We do not even know the energy source of GRBs. We do know that GRBs are far from equilibrium, evolving explosively on a time scale of order $10^{-3} \mathrm{~s}$.

We want to do extragalactic neutrino astronomy because it is truly an exploration of the universe. We do solar neutrino astronomy to test fundamental theories of physics and astronomy. But, perhaps solar neutrino research and extragalactic neutrino research may in the end share a fundamental characteristic: surprise. Remember, that we undertook solar neutrino research to test stellar evolution and unexpectedly (at least for everybody except Bruno Pontecorvo) we found evidence for new neutrino physics.

In a sense, we are returning to our original goal in neutrino astronomy, but by a round-about 
path. We must first understand neutrino oscillation phenomena in order to be able to use solar neutrino observations to test precisely the theory of stellar evolution, our original goal. Perhaps with extragalactic astronomy we will participate in a similar cycle of astronomical exploration and physical clarification. T. S. Elliot in 'The Four Quartets' described the cycle succinctly and beautifully:

We shall not cease from exploration

And the end of all of our exploring

Will be to arrive where we started

And know the place for the first time.

\section{Acknowledgments}

I acknowledge support from NSF grant \#PHY95-13835. 